\title{
Nalbuphine analgesic and anti-inflammatory effects on patients undergoing thoracoscopic lobectomy during the perioperative period
}

\author{
YANG ZHANG ${ }^{1 *}$, QI JIANG ${ }^{1 *}$ and TAO $\mathrm{LI}^{2}$ \\ ${ }^{1}$ Department of Anesthesiology, Xiangyang No. 1 People's Hospital, Hubei University of Medicine, Xiangyang, Hubei 441000; \\ ${ }^{2}$ Department of Anesthesiology, China-Japan Union Hospital of Jilin University, Changchun, Jilin 130033, P.R. China
}

Received May 24, 2017; Accepted August 3, 2017

DOI: $10.3892 /$ etm.2017.4920

\begin{abstract}
This study sought to investigate the analgesic effects of nalbuphine on patients undergoing thoracoscopic lobectomy during the perioperative period, as well as its effects on inflammatory cytokines. We selected 92 patients with early lung cancer who were admitted to Xiangyang No. 1 People's Hospital between January 2016 and December 2016. The patients were randomly divided into control and observation groups ( $\mathrm{n}=46$ each). All patients underwent thoracoscopic lobectomy, with those in the observation group receiving intravenous nalbuphine hydrochloride prior to induction (the control group received saline). Intraoperative blood loss, operation time and anesthetic dosages were compared between groups. The analgesic effects during the perioperative period were compared using a visual analogue scale. The adverse effects of anesthetics (nausea, vomiting, dizziness and drowsiness) were compared between the two groups. Finally, serum inflammatory cytokine [tumor necrosis factor- $\alpha$ (TNF- $\alpha$ ), interleukin-6 (IL-6) and IL-10] levels were measured using ELISA at 1, 3, 5 and 7 days after operation. There were no significant differences in intraoperative blood loss and operation time between the two groups ( $\mathrm{p}>0.05)$. However, dosages of propofol and remifentanil used were lower in the observation group than in the control group $(\mathrm{p}<0.05)$, and the effective rate of postoperative analgesia in the observation group was significantly higher than that in the control group $(\mathrm{p}<0.05)$. The degree of postoperative pain in both groups were lower than preoperative rates $(\mathrm{p}<0.05)$, while the incidence of adverse reactions in the observation group was significantly lower than
\end{abstract}

Correspondence to: Dr Tao Li, Department of Anesthesiology, China-Japan Union Hospital of Jilin University, 126 Xiantai Street, Changchun, Jilin 130033, P.R. China

E-mail: ib248u@163.com

*Contributed equally

Key words: thoracoscopic lobectomy, lung cancer, nalbuphine, analgesic effect, inflammatory cytokines in the control group $(\mathrm{p}<0.05)$. Finally, TNF- $\alpha$, IL-6 and IL-10 levels were significantly lower in the observation group relative to the control group at $1,3,5$ and 7 days after operation $(\mathrm{p}<0.05)$. Therefore, nalbuphine has a significant analgesic effect during thoracoscopic lobectomy. The application of nalbuphine can reduce the incidence of adverse reactions, reduce postoperative inflammatory responses, and promote rapid patient recovery, thus demonstrating the clinical value of nalbuphine.

\section{Introduction}

Due to environmental pollution caused by industrialization, tobacco exposure, automobile exhaust pollution, host characteristics, and other factors, lung cancer cases are increasing and has become one of the most common causes of cancer-related death (1). In China, 600,000 people die each year from lung cancer and lung cancer mortality ranked first among all malignant tumors (2). Surgery is usually characterized by severe traumatic events and long operation times. Anesthetic-induced stimulation can lead to stress and inflammatory responses, neither of which being conducive to a good prognosis (3). With the continuous development of minimally invasive technology, thoracoscopic lobectomy is now well-accepted for the early treatment of lung cancer by clinicians and patients, as it presents reduced surgical trauma and quicker recoveries (4). Therefore, anesthesia-induced inflammatory response, postoperative pain, and the incidence of adverse reactions should be reduced in order to improve patient recovery, shorten hospital stays, and reduce patient economic burdens (5). Nalbuphine is an opioid analgesic with a good analgesic effect (6). In this study, patients undergoing thoracoscopic lobectomy were treated with nalbuphine to explore its analgesic properties and effects on inflammatory cytokines, in order to identify a more suitable anesthesia program for patients undergoing early lung cancer surgery.

\section{Materials and methods}

General patient information. Ninety-two patients with early lung cancer admitted to Xiangyang No. 1 People's Hospital between January 2016 and December 2016 were selected. Patients were randomly assigned to control and observation groups ( $\mathrm{n}=46$ each). This study was approved by the Ethics 
Committee of Xiangyang No. 1 People's Hospital. Signed written informed consents were obtained from the patients and/or guardians. Inclusion criteria were as follows: Presenting with lung cancer as diagnosed by imaging and pathological examinations, willing to receive thoracoscopic lobectomy and signed informed consent. Exclusion criteria were as follows: Presenting abnormal coagulation, presenting a history of thoracotomy, hilar and mediastinal lymph node metastasis. There were no significant differences in general characteristics between the two groups ( $p>0.05$; Table I).

\section{Methods}

Preoperative preparation. Both groups were subjected to routine preoperative examinations, and any patients presenting surgical contraindications were excluded. Patients were asked to quit smoking 2 weeks before surgery and patients fasted for $6 \mathrm{~h}$ before surgery.

Anesthesia. Patient arterial blood pressure, pulse and electrocardiograms were monitored after entering the operating room. Prior to the induction of anesthesia, patients in the observation group received $20 \mathrm{mg}$ of nalbuphine (SFDA approval no. 20130127; Yichang Renfu Pharmaceutical Co., Ltd., Yichang, China) intravenously, while control group patients received the same amount of $9 \%$ saline. A single injection of fentanyl ( $3 \mu \mathrm{g} / \mathrm{kg}$, SFDA no. H42022076; Yichang Renfu Pharmaceutical Co., Ltd.) was performed. Intravenous injection of propofol with micropump was administered (SFDA no. H20084531; Zhejiang Jiuxu Pharmaceutical Co., Ltd., Zhejiang, China) with a target concentration of $3 \mu \mathrm{g} / \mathrm{ml}$. A single injection of cisatracurium $(0.02 \mathrm{mg} / \mathrm{kg}$, SFDA no. H20060869; Jiangsu Hengrui Medicine Co., Ltd., Jiangsu, China) was administered for the induction of anesthesia. Tracheal intubation was performed with an EEG bispectral index value below 55. Combined intravenous-inhalation anesthesia was administered by continuous infusion of remifentanil (SFDA no. H20143314; Jiangsu Nhwa Pharmaceutical Co., Ltd., Jiangsu, China) and propofol, with intermittent infusion of cisatracurium.

Surgical approach. Both groups of patients underwent video-assisted thoracoscopic lobectomy. Patients were in the lateral position. After conventional disinfection, an incision was made between the 7 and 8th ribs along the midaxillary line to serve as an observation hole, with another incision made between the 4 and 5th ribs along the anterior axillary line to be used as the main operating hole. A third incision was made between the 8 and 9 th rib along the subscapularis line to serve as a secondary operating hole. Thoracoscopy was performed to check the thoracic cavity. Upper left lung segment, tongue segment, right upper lung anterior segment, right upper lung tip, dorsal segment, right upper lung posterior segment or other lung segments were resected according to disease conditions. Mediastinal lymph nodes were dissected and lung swelling was examined. Lung and bronchial stump leakage were also examined. Chest tube was then placed using the observation tube and the incision was sutured.

Inflammatory index examination. Arterial blood (5 ml) was extracted at 1,3,5 and 7 days after operation. Serum levels of tumor necrosis factor- $\alpha$ (TNF- $\alpha$ ), interleukin-6 (IL-6) and IL-10 were measured by enzyme-linked immunosorbent assay (ELISA) using a kit from Beckman Coulter, Inc. (Brea, CA,
Table I. General patient characteristics.

\begin{tabular}{lcccc}
\hline Characteristics & $\begin{array}{c}\text { Control } \\
\text { group }(\mathrm{n}=46)\end{array}$ & $\begin{array}{c}\text { Observation } \\
\text { group }(\mathrm{n}=46)\end{array}$ & $\mathrm{t} / \chi^{2}$ & P-value \\
\hline Sex (male/female) & $29 / 17$ & $27 / 19$ & 0.046 & 0.831 \\
Age (years) & $40-70$ & $40-75$ & & \\
$\begin{array}{l}\text { Average age } \\
\text { (years) }\end{array}$ & $56.48 \pm 7.56$ & $57.23 \pm 8.63$ & 0.443 & 0.659 \\
BMI $\left(\mathrm{kg} / \mathrm{m}^{2}\right)$ & $21.69 \pm 3.49$ & $21.83 \pm 3.58$ & 0.190 & 0.849 \\
Smoking $(\mathrm{n}, \%)$ & & & & \\
$\geq 5$ cigarettes & $21(5.65)$ & $18(39.13)$ & 0.508 & 0.776 \\
$<5$ cigarettes & $7(15.22)$ & $9(19.57)$ & & \\
Non-smoking & $18(39.13)$ & $19(41.30)$ & & \\
& & & &
\end{tabular}

USA). All operations were performed in strict accordance with manufacturer's instructions. Optical density (OD) values at $450 \mathrm{~nm}$ were measured by a microplate reader (Potebio, Jiangsu, China) and were used to calculate TNF- $\alpha$, IL-6 and IL-10 levels.

\section{Evaluation criteria}

Operation time, intraoperative blood loss, and propofol and remifentanil dosages were recorded for both groups. Postoperative adverse reactions caused by anesthetics, including nausea, vomiting, dizziness and drowsiness, were also recorded.

Visual analogue scale (VAS). A VAS was used to assess analgesic effects. The scores ranged from 0 to 10 (0 points representing painless and 10 points indicating unbearable severe pain). Evaluation criteria were as follows: Level I: 0-2 points, painless or slight pain, excellent analgesic effect; level II: 3-5 points, tolerable pain, good analgesic effect; level III: 6-8 points, obvious pain, poor analgesic effect; and level IV: $\geq 8$ points, intolerable pain, no analgesic effect. Analgesic efficacy $=($ level I + II $) /$ no. of patients x $100 \%$.

Post-operative serum inflammation index analysis. Fasting venous blood $(2-5 \mathrm{ml})$ was collected from patients in the morning. Serum inflammatory factors levels, including TNF- $\alpha$, IL- 6 and IL-10, were measured by ELISA.

Statistical analysis. Data were processed using SPSS 19.0 statistical software (SPSS Inc., Chicago, IL, USA). Measurement data are expressed as mean \pm standard deviation, and t-test was used for comparisons. Count data were expressed as rates and $\chi^{2}$ test was performed for comparisons. $\mathrm{P}<0.05$ was considered to indicate a statistically significant difference.

\section{Results}

Comparison of surgical conditions and anesthesia effects. There were no significant differences in operation time and intraoperative blood loss between the two groups. Propofol and remifentanil dosages required by the observation group were significantly lower than the control group ( $\mathrm{p}<0.05$; Table II).

Comparison of analgesic effects. The effective rate of analgesia in the observation group was significantly higher than in 
Table II. Comparison of surgical conditions and anesthesia effects.

\begin{tabular}{|c|c|c|c|c|c|}
\hline Groups & Cases & $\begin{array}{l}\text { Operation time } \\
\quad(\min )\end{array}$ & $\begin{array}{l}\text { Intraoperative } \\
\text { blood loss }(\mathrm{ml})\end{array}$ & $\begin{array}{c}\text { Propofol } \\
\text { dosage (mg) }\end{array}$ & $\begin{array}{l}\text { Remifentanil } \\
\text { dosage }(\mu \mathrm{g})\end{array}$ \\
\hline Observation & 46 & $124.76 \pm 9.15$ & $226.13 \pm 9.63$ & $1084.23 \pm 21.53$ & $2204.23 \pm 71.53$ \\
\hline Control & 46 & $125.34 \pm 9.77$ & $225.87 \pm 9.38$ & $1467.56 \pm 22.47$ & $2977.56 \pm 72.48$ \\
\hline t-test & & 1.974 & 0.131 & 85.544 & 51.506 \\
\hline P-value & & 0.769 & 0.896 & $<0.001$ & $<0.001$ \\
\hline
\end{tabular}

Table III. Comparison of analgesic effects (n, \%).

\begin{tabular}{|c|c|c|c|c|c|c|}
\hline Groups & Cases & Level I & Level II & Level III & Level IV & Effective rate of analgesia \\
\hline Observation & 46 & $36(78.26)$ & $7(15.22)$ & $3(6.52)$ & $0(0.00)$ & $43(93.47)$ \\
\hline Control & 46 & $18(39.13)$ & $14(30.43)$ & $11(23.91)$ & $3(6.52)$ & $32(69.56)$ \\
\hline & & & & & & 15.905 \\
\hline P-value & & & & & & 0.001 \\
\hline
\end{tabular}

Table IV. Comparison of adverse effects caused by anesthetics (n, \%).

\begin{tabular}{|c|c|c|c|c|c|c|}
\hline Groups & Cases & Nausea & Vomiting & Dizziness & Drowsiness & All adverse effects \\
\hline Observation & 46 & $3(6.52)$ & $1(2.17)$ & $1(2.17)$ & $1(2.17)$ & $6(13.04)$ \\
\hline Control & 46 & $6(13.04)$ & $4(8.70)$ & $5(10.87)$ & $5(10.86)$ & $20(43.48)$ \\
\hline$\chi^{2}$ & & & & & & 9.061 \\
\hline P-value & & & & & & 0.003 \\
\hline
\end{tabular}

Table V. Comparison of TNF- $\alpha$ levels at different time-points (ng/l).

\begin{tabular}{|c|c|c|c|c|c|}
\hline Groups & Cases & Day 1 & Day 3 & Day 5 & Day 7 \\
\hline Observation & 46 & $64.19 \pm 5.43$ & $98.73 \pm 6.14$ & $44.23 \pm 5.28$ & $28.78 \pm 4.16$ \\
\hline Control & 46 & $73.34 \pm 5.74$ & $112.25 \pm 6.65$ & $61.75 \pm 5.19$ & $39.56 \pm 4.43$ \\
\hline t-test & & 7.854 & 10.131 & 16.050 & 12.031 \\
\hline P-value & & $<0.001$ & $<0.001$ & $<0.001$ & $<0.001$ \\
\hline
\end{tabular}

TNF- $\alpha$, tumor necrosis factor- $\alpha$.

the control group $(\mathrm{p}<0.05)$. Therefore, pain was significantly reduced in observation group $(\mathrm{p}<0.05$; Table III).

Comparison of adverse effects caused by anesthetics. Nausea, vomiting, dizziness and drowsiness were found in 6 patients in the observation group (incidence rate 13.04\%). Those adverse effects were found in 20 patients in the control group (incidence rate $43.48 \%$ ). The adverse effect incidence rate was therefore significantly lower in the observation group compared to the control group ( $\mathrm{p}<0.05$; Table IV).

Comparison of TNF- $\alpha, I L-6$ and IL-10 levels. The levels of TNF- $\alpha$, IL- 6 and IL-10 in the observation group were significantly lower than in the control group ( $\mathrm{p}<0.05$; Tables V-VII).

\section{Discussion}

At present, the morbidity and mortality of lung cancer is showing an increasing trend, and lung cancer has become a principle killer (7). Early clinical manifestations of lung cancer are not obvious, and advanced lung cancer can be characterized by weight loss, fever, cough, chest tightness, shortness of breath, chest pain, bloody sputum and hemoptysis (8). Advanced lung cancer is usually accompanied by metastasis, leading to poor prognosis and low survival rates (9). Surgery is the preferred treatment for lung cancer. Compared with traditional thoracic surgery, thoracoscopic lobectomy can reduce surgical trauma, and the visual field can be amplified by video, allowing for the clearer visualization of lymph nodes and small blood 
Table VI. Comparison of IL-6 levels at different time-points (ng/l).

\begin{tabular}{lccccc}
\hline Groups & Cases & Day 1 & Day 3 & Day 5 & Day 7 \\
\hline Observation & 46 & $126.49 \pm 6.68$ & $153.03 \pm 8.16$ & $98.53 \pm 6.78$ & $52.65 \pm 5.26$ \\
Control & 46 & $153.36 \pm 6.77$ & $176.54 \pm 8.74$ & $129.62 \pm 6.87$ & 21.864 \\
t-test & & 19.162 & 13.355 & $<0.001$ & 21.030 \\
P-value & & $<0.001$ & $<0.001$ & $<0.001$ \\
\hline
\end{tabular}

IL-6, interleukin-6.

Table VII. Comparison of IL-10 levels at different time-points (ng/l).

\begin{tabular}{lccccc}
\hline Groups & Cases & Day 1 & Day 3 & Day 5 & Day 7 \\
\hline Observation & 46 & $157.68 \pm 7.43$ & $182.34 \pm 9.13$ & $124.39 \pm 8.28$ & $65.72 \pm 5.63$ \\
Control & 46 & $198.18 \pm 7.92$ & $213.76 \pm 9.68$ & $157.65 \pm 8.57$ & $82.93 \pm 6.67$ \\
t-test & & 25.294 & 16.015 & 18.930 & 13.373 \\
P-value & & $<0.001$ & $<0.001$ & $<0.001$ & $<0.001$ \\
\hline
\end{tabular}

IL-10, interleukin-10.

vessels and reducing unnecessary damage. In addition, the separation and ligation of fine structures can be achieved and the efficiency of lymph node dissection is considerable $(10,11)$.

Nalbuphine is a type of agonist-antagonist morphine drug with analgesic and sedative effects which was first used clinically in the 1970s (12). This study found no significant differences in terms of operation time and intraoperative blood loss between the observation and control groups. However, propofol and remifentanil dosages required in the observation group were significantly lower than in the control group. The effective rate of analgesia was significantly increased and the degree of pain was significantly reduced in the observation group relative to controls as well $(\mathrm{p}<0.05)$. This indicates that the combined effects of nalbuphine, propofol and remifentanil can significantly increase the effect of analgesia, and dosages of propofol and remifentanil can be reduced without affecting the anesthetic effect. We also found that the incidence of adverse reactions such as nausea, vomiting, dizziness and drowsiness was significantly lower in observation group $(\mathrm{p}<0.05)$. Adverse reactions caused by anesthetics are usually mediated by $\mu$ receptors. Propofol and remifentanil, which are $\mu$ receptor agonist-antagonists, can cause nausea, vomiting and other adverse reactions (13). In contrast, nalbuphine can antagonize $\mu$ receptors and impart decreased impact on hemodynamics in order to avoid tachycardia. In addition, the inhibitory effect of nalbuphine on respiration is slight and has capping effect (14). Nalbuphine is also agonistic to $\kappa$ receptors, and $\kappa$ receptors are present in the peripheral and central nervous systems. Agitation of $\kappa$ receptors can lead to strong and persistent analgesia, which in turn reduces the incidence of adverse effects caused by propofol and remifentanil (15).

Lung cancer surgery and anesthesia can both cause stress responses in patients affecting catechol levels, which in turn stimulates the secretion of inflammatory cytokines and causes inflammation (16). TNF- $\alpha$, which is mainly produced by macrophages, NK cells and T lymphocytes, can initiate and trigger inflammation, which in turn leads to tumor cell necrosis and anti-infection immune functions (17). IL-6, which is an acute phase response lymphocytic factor, is a member of the IL family, which plays important roles in the immune response (18). Another member of interleukin family, IL-10, can inhibit the activity of natural killer cells and depress immune system tumor cell monitoring. Under normal conditions, the expression level of IL-10 is low, but its expression can be significantly increased by inflammation (19). In this study, TNF- $\alpha$, IL-6 and IL-10 levels in the observation group were significantly lower than in the control group at 1,3,5 and 7 days post-operation $(\mathrm{p}<0.05)$. Inflammatory cytokine levels peaked on day 3 and started to decline at day 5. Stimulation caused by surgical trauma and anesthetics can lead to a series of stress responses in the body, leading to significantly increased secretion of adrenaline and catechol, which in turn led to increased postoperative levels of TNF- $\alpha$, IL-6 and IL-10. Nalbuphine can increase the analgesic effect so that traumatic stimulation of the central nerve system can be effectively blocked, thereby reducing the stress response and affecting immune function regulation. Stress response hormone secretion, such as plasma cortisol and catecholamines, can be reduced, and macrophage activation can be inhibited to achieve anti-sympathetic, sedative and antioxidant effects. Therefore, the peripheral and central nervous system sensitization will be reduced and IL-10, TNF- $\alpha$ and IL-6 expression will be downregulated as part of an anti-inflammatory effect (20).

In conclusion, the intravenous injection of nalbuphine prior to induction of anesthesia during thoracoscopic lobectomy can effectively relieve perioperative pain and reduce inflammatory responses in patients with lung cancer, thus promoting recovery. 


\section{References}

1. Cooper WA: Preface-molecular genetics of lung cancer. Transl Lung Cancer Res 4: 109, 2015.

2. Niu X and Wang Q: MicroRNA and lung cancer. Zhongguo Fei Ai Za Zhi 13: 380-385, 2010 (In Chinese).

3. Sprung J, Scavonetto F, Yeoh TY, Kramer JM, Karnes RJ, Eisenach JH, Schroeder DR and Weingarten TN: Outcomes after radical prostatectomy for cancer: A comparison between general anesthesia and epidural anesthesia with fentanyl analgesia: A matched cohort study. Anesth Analg 119: 859-866, 2014.

4. Yu PS, Capili F and Ng CS: Single port VATS: Recent developments in Asia. J Thorac Dis 8 (Suppl 3): S302-S307, 2016.

5. Manchikanti L, Cash KA, Pampati V, Wargo BW and Malla Y: Management of chronic pain of cervical disc herniation and radiculitis with fluoroscopic cervical interlaminar epidural injections. Int J Med Sci 9: 424-434, 2012.

6. Chatrath V, Attri JP, Bala A, Khetarpal R, Ahuja D and Kaur S: Epidural nalbuphine for postoperative analgesia in orthopedic surgery. Anesth Essays Res 9: 326-330, 2015.

7. Travis WD, Travis LB and Devesa SS: Lung cancer. Cancer 75 (Suppl 1): 191-202, 1995.

8. Lin L, Gu ZT, Chen WH and Cao KJ: Increased expression of the long non-coding RNA ANRIL promotes lung cancer cell metastasis and correlates with poor prognosis. Diagn Pathol 10 14-21, 2015.

9. Mohamed SA, Mousa EM, Hamed AM, Amin SE and Aziz N: Utility of multidetector row computed tomography and virtual bronchoscopy in evaluation of hemoptysis due to lung cancer. Egypt J Chest Dis Tuberc 65: 279-287, 2016.

10. Liu C, Li Z, Bai C, Wang L, Shi X and Song Y: Video-assisted thoracoscopic surgery and thoracotomy during lobectomy for clinical stage I non-small-cell lung cancer have equivalent oncological outcomes: A single-center experience of 212 consecutive resections. Oncol Lett 9: 1364-1372, 2015.

11. Goto T, Kadota Y, Mori T, Yamashita S, Horio H, Nagayasu T and Iwasaki A: Video-assisted thoracic surgery for pneumothorax: Republication of a systematic review and a proposal by the guideline committee of the Japanese Association for Chest Surgery 2014. Gen Thorac Cardiovasc Surg 63: 8-13, 2015.
12. Fating DR, Dandekar AK and Tirpude NG: Role of IV nalbuphine in attenuation of haemodynamic response to laryngoscopy and endotracheal intubation. Indian J Clin Anaesth 3: 165-169, 2016.

13. Hu LG, Pan JH, Li J, Kang F and Jiang L: Effects of different doses of sufentanil and remifentanil combined with propofol in target-controlled infusion on stress reaction in elderly patients. Exp Ther Med 5: 807-812, 2013.

14. Zeng Z, Lu J, Shu C, Chen Y, Guo T, Wu QP, Yao SL and Yin P: A comparision of nalbuphine with morphine for analgesic effects and safety: Meta-analysis of randomized controlled trials. Sci Rep 5: 10927, 2015.

15. Jannuzzi RG: Nalbuphine for treatment of opioid-induced pruritus: A systematic review of literature. Clin J Pain 32: 87-93, 2016.

16. Hong Y and Rurong W: Systemic and alveolar inflammatory response in the dependent and nondependent lung in patients undergoing lung resection surgery. Eur J Anaesthesiol 33: 63-64, 2016.

17. Murdaca G, Spanò F, Contatore M, Guastalla A, Penza E, Magnani $\mathrm{O}$ and Puppo F: Infection risk associated with anti-TNF- $\alpha$ agents: A review. Expert Opin Drug Saf 14: 571-582, 2015.

18. Pedersen BK and Febbraio M: Exercise and interleukin- 6 action. Expert Rev Endocrinol Metab 2014: 319-321, 2014.

19. Zhang YM, Mao YM and Sun YX: Genetic polymorphisms of IL-6 and IL-10 genes correlate with lung cancer in neversmoking Han population in China. Int J Clin Exp Med 8: 1051-1058, 2015

20. Purdy M,Kokki M, Anttila M, Aspinen S, Juvonen P, Korhonen R, Selander T, Kokki H and Eskelinen M: Does the rectus sheath block analgesia reduce the inflammatory response biomarkers' IL-1ra, IL-6, IL-8, IL-10 and IL-1 $\beta$ concentrations following surgery? A randomized clinical trial of patients with cancer and benign disease. Anticancer Res 36: 3005-3011, 2016. 